\title{
Conservation of the global sex determination gene tra-1 in distantly related nematodes
}

\author{
André Pires-daSilva and Ralf J. Sommer ${ }^{1}$ \\ Max-Planck Institut für Entwicklungsbiologie, Abteilung Evolutionsbiologie, Spemannstrasse 37-39, \\ D-72076 Tübingen, Germany
}

\begin{abstract}
Sex determination has long intrigued evolutionists, geneticists, and developmental biologists in a similar way. Substantial evidence indicates that sex determination evolves rapidly and, therefore, can be used to study how molecular patterning processes evolve. In Caenorhabditis elegans, sex determination relies on a signaling pathway that involves a cascade of negatively acting factors, finally triggering the GLI-family zinc-finger transcription factor TRA-1. We have started to investigate sex determination in the nematode satellite species Pristionchus pacificus that is separated from C. elegans for 200-300 million years. In P. pacificus, animals with two X chromosomes develop as hermaphrodites, whereas XO animals develop as males. We used an unbiased forward genetic approach and isolated several mutants with a hermaphrodite to male transformation of the XX karyotype. We identified one complementation group as representing the $P$. pacificus ortholog of tra-1, providing the first evidence for the conservation of a global sex determination gene over a time period of at least 200 million years. A Ppa-tra-1 morpholino phenocopies Ppa-tra-1 mutants and establishes the morpholino technology as a reverse genetic approach in $P$. pacificus.
\end{abstract}

[Keywords: Sex determination; evolution; P. pacificus; C. elegans; tra-1]

Received December 1, 2003; revised version accepted March 26, 2004.

Signaling pathways are crucial for the cell-cell interactions that orchestrate animal development (for review, see Gerhart 1999). The activation of a signaling pathway usually starts with the binding of a ligand to a transmembrane protein, which transmits the signal via a cascade of transducers to the nucleus. The ultimate consequence of such a cascade is the regulation of gene expression in a group of cells, affecting their fate, movement, and/or shape. Biochemical and genetic analyses have uncovered a multitude of genetic interactions and components that constitute the different signaling pathways (for review, see Barolo and Posakony 2002).

Surprisingly, most metazoans share a common set of signaling pathways, despite their different modes of development and body plans (for review, see Pires-daSilva and Sommer 2003). Most cell-cell interactions during development rely on Hedgehog, Wnt, TGF $\beta$, receptortyrosine kinase, Notch, JAK/STAT, and nuclear hormone pathways. The comparison of signaling systems in different organisms can help to elucidate how pathways evolve. Do new types of connectivity arise between the same sets of genes? Are gene replacement, gene loss, or

${ }^{1}$ Corresponding author.

E-MAIL ralf.sommer@tuebingen.mpg.de; FAX 49-7071-601-498. Article and publication are at http://www.genesdev.org/cgi/doi/10.1101/ $\operatorname{gad} .293504$. gene addition of importance during the evolution of signaling pathways? Comparing insects, vertebrates, nematodes, and cnidarians can provide answers to such questions. However, it is more difficult to reconstruct the events that led to the first appearance of the seven primary signaling pathways when interphyletic comparisons are made. Instead, studying fast evolving signaling pathways in more closely related species, preferentially within one phylum, might be a more fruitful approach to obtain insight into the evolution of signaling pathways (Pires-daSilva and Sommer 2003).

An example of a fast evolving pathway is the sex determination pathway (Bull 1983; Wilkins 1995; Marin and Baker 1998). For instance, no similarities have been observed between Drosophila and Caenorhabditis elegans sex determination genes, except for the recent identification of the downstream transcription factors doublesex and mab-3 (Raymond et al. 1998). The housefly Musca domestica and the fruitfly Drosophila that shared a last common ancestor around 100 million years ago (mya), have only one common gene in their sex determination pathways (Hediger et al. 2004). In several other cases, differences in sex determination mechanisms exist among even more closely related species, sometimes involving environmental cues (Pieau 1996).

One species in which the genetics and molecular biology of sex determination have been studied in detail is the nematode model organism C. elegans (for recent re- 
view, see Goodwin and Ellis 2002). The detailed knowledge of the $C$. elegans sex determination mechanism provides a framework for comparative studies in other nematode species (Stothard and Pilgrim 2003). The primary signal of $C$. elegans sex determination is the ratio of the set of X chromosomes to autosomes. Essentially, animals with a single $\mathrm{X}$ chromosome develop as males (XO), whereas XX animals develop as hermaphrodites. Several mutations with a sex reversal have been described. Mutations in the transformer loci result in XX animals, which develop as pseudomales (Hodgkin and Brenner 1977). On the contrary, mutations in the fem or her genes result in $\mathrm{XO}$ animals developing as females and hermaphrodites, respectively (Nelson et al. 1978; Hodgkin 1980). Genetic epistasis analyses of sex-reversed mutants have placed the $C$. elegans sex determination genes in a regulatory pathway (Fig. 1A). A high $\mathrm{X}$-chromosome dose results in the activation of the transcription factor TRA-1 in the hermaphroditic soma. In males, which have a low X-chromosome dose, the FEM proteins inhibit TRA-1 activity (Fig. 1A,B).

Generally, the regulation of sex determination can be divided into two phases. In the initial phase, "global" sex determination regulators control the sex of the complete organism. For instance, all of the sex determination genes of $C$. elegans acting upstream of tra-1 are globally

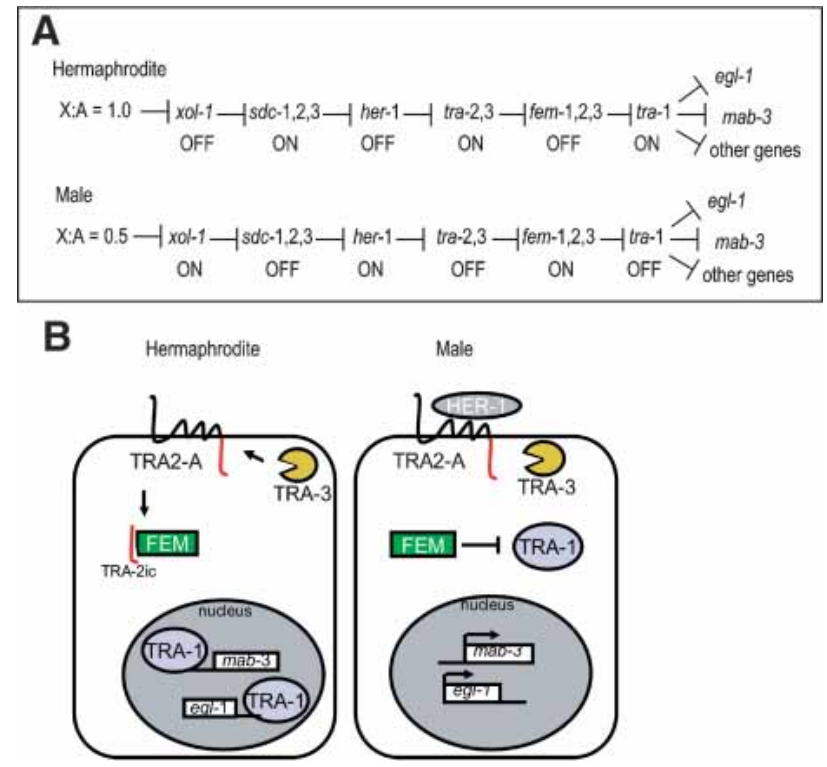

Figure 1. Genetic $(A)$ and molecular $(B)$ model for sex determination in C. elegans. (A) The ratio of $\mathrm{X}$ chromosomes to the set of autosomes determines whether an animal develops as hermaphrodite or male. In animals with a 1:0 ratio, a cascade of negative interactions results in the activation of TRA-1, promoting hermaphroditic development. In males, TRA-1 is inactive. (B) In hermaphrodites, TRA-3 cleaves the intracellular domain of TRA-2 (TRA-2ic), which inhibits the FEM proteins. As a result, TRA-1 is translocated to the nucleus and regulates target genes. In males, HER-1 inhibits the cleavage of TRA-2 by TRA-3. As a result, TRA-1 is retained in the cytoplasm by the FEM proteins. acting factors. If a gene encoding a global regulator is mutated, the sex of the organism is completely transformed. Factors that act more downstream in the genetic hierarchy are "local" sex determination genes. In the case of C. elegans, several transcriptional targets of tra-1, such as egl-1, mab-3, or fog-3, have been identified that regulate sex locally, in a tissue or cell-specific manner (Trent et al. 1983; Shen and Hodgkin 1988; Ellis and Horvitz 1991; Ellis and Kimble 1995).

Within the phylum Nematoda, comparative studies have so far been limited to the genus Caenorhabditis. These studies show a dual pattern. The sequences of sex determination genes evolved rapidly, whereas the function of all of those genes studied to date are conserved. HER-1, FEM-2, FEM-3, TRA-2, and TRA-1 are among the fastest evolving proteins within the genus /de Bono and Hodgkin 1996; Kuwabara 1996; Hansen and Pilgrim 1998; Streit et al. 1999; Haag et al. 2002). They show an overall amino acid identity that ranges from $37 \%$ to $63 \%$ between $C$. elegans and $C$ briggsae, although both species shared a common ancestor around 100 mya. However, the poor sequence conservation of sex determination genes in Caenorhabditis is in contrast with their functional conservation. Gene-silencing studies using RNA-mediated interference (RNAi) or transgenic rescue experiments suggest that many of the genes involved in the somatic sex determination of $C$. elegans are conserved between C. elegans, C. briggsae, and C. remanei. In C. briggsae, RNAi -experiments with Cbr-fem-1, Cbrfem-2, Cbr-fem-3, Cbr-her-1, and Cbr-tra-2 produce similar sex-reversal effects to those known from C. elegans orthologous mutants (Hansen and Pilgrim 1998; Streit et al. 1999; Haag and Kimble 2000; Haag et al. 2002; Stothard et al. 2002; Stothard and Pilgrim 2003). Similar results were found in C. remanei for Cre-fem-3 and Cre-tra-2 and for fem-1 in C. sp (CB5161; Haag and Kimble 2000; Haag et al. 2002; Stothard et al. 2002). As an independent assay to determine the conservation of sex determination genes, rescue experiments using $C$. elegans mutants were performed: Cbr-tra-1, Cbr-her-1, Cbr-fem-2, Cbr-fem-1, and Cs(CB5161)-fem-2 rescued the phenotype of the respective $C$. elegans mutant /de Bono and Hodgkin 1996; Hansen and Pilgrim 1998; Streit et al. 1999; Stothard et al. 2002; Stothard and Pilgrim 2003).

To study the evolution of signaling pathways, we decided to compare nematode sex determination in more distantly related species, using an unbiased forward genetic approach in the nematode Pristionchus pacificus, a member of the Diplogastridae family. P. pacificus and $C$. elegans shared the last common ancestor around 200300 mya. $P$. pacificus has been best characterized for its vulva development, which shows many differences at the cellular and genetic level when compared with $C$. elegans (for review, see Sommer 2001). P. pacificus also develops into either hermaphrodite or male and has life history characteristics that are of advantage for genetic studies, such as a short life cycle and large brood sizes (Sommer et al. 1996). Furthermore, the availability of an integrated physical and genetic map enhances positional 
cloning of induced mutations (Srinivasan et al. 2002, 2003).

Here, we describe a screen for sexual transformation in $P$. pacificus. We show that mutants with a hermaphrodite to male transformation are caused by mutations in the $P$. pacificus tra-1 gene, providing the first evidence for the conservation of a global sex determination gene over a time period of at least 200 million years. Morpholino knockdown of Ppa-tra-1 leads to a similar phenotype as Ppa-tra-1 mutants and result in a nearly complete sex reversal in the soma. Like Cel-tra-1, Ppa-tra-1 mutants generate some oocytes indicating that the germ line transformation is incomplete.

\section{Results}

Background: Genetics of sex determination in C. elegans

In C. elegans, many mutations causing sexual transformation phenotypes have been isolated. tra-1, tra-2, and tra-3 transform genotypic hermaphrodites into phenotypic males (Hodgkin and Brenner 1977; Hodgkin 1980). Many alleles of tra-1 and tra-2 have been found, some of which produce an almost complete transformation into fertile males. In contrast, tra-3 alleles are rare and show a maternal effect (Hodgkin 1980). Mutations in the fem genes result in both XX and XO animals exhibiting female phenotypes (Nelson et al. 1978; Hodgkin 1980), and her-1 mutations result in XO animals showing hermaphroditic fates (Hodgkin 1980). Epistasis analysis indicated these sex determination genes act as a cascade of negative interactors. tra-1 is the most downstream gene and suppresses male fates in XX animals. It encodes a transcription factor of the $\mathrm{C} 2 \mathrm{H} 2$ zinc-finger class and belongs to the Gli/CiD family of proteins (Zarkower and Hodgkin 1992). In tra-1 (loss-of-function) mutants, XX animals are transformed into males.

\section{A screen for sexual transformers in P. pacificus}

In laboratory stocks of $P$. pacificus var. California, males occur at a frequency of about $0.1 \%$ as the result of meiotic nondisjunction of the $\mathrm{X}$ chromosome (Sommer et al. 1996, Srinivasan et al. 2002). To identify P. pacificus sex determination mutants, we performed an $F_{1}$ clonal screen for transformation of XX hermaphrodites into phenotypic males (Fig. 2A). From an EMS mutagenesis screen of $\sim 7000$ haploid genomes, several putative sex determination mutants showed a high frequency of males in the progeny of clonal $\mathrm{F}^{1}$ animals. All such candidate mutants were crossed with the polymorphic $P$. pacificus strain var. Washington to determine whether these phenotypic males have an XX genotype. Using the $\mathrm{X}$ chromosome-linked single-strand conformation polymorphism (SSCP) markers S105 and S140 (Srinivasan et al. 2002), we found that some of these males were indeed heterozygous for the $\mathrm{X}$ chromosome (Fig. 2B). These results indicate that these mutants are sex determination defective, rather than mutants with an elevated level of $\mathrm{X}$-chromosomal nondisjunction.
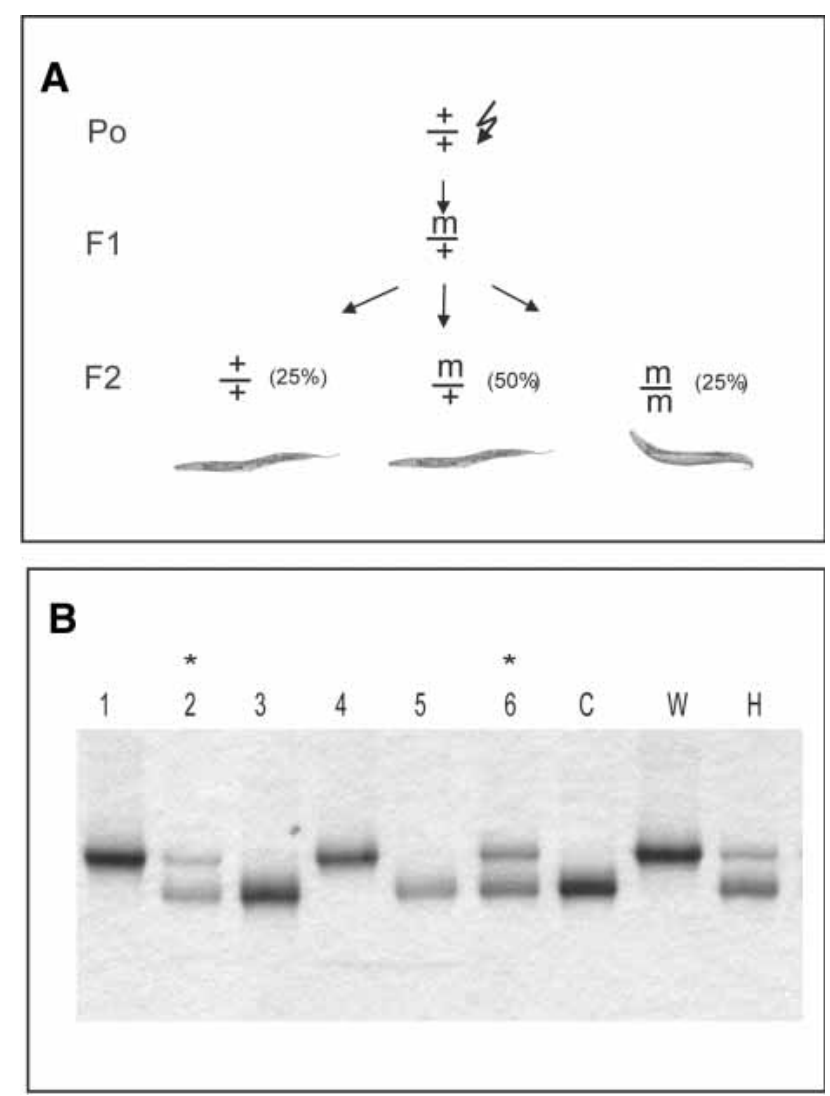

Figure 2. Identification of sex determination mutants. (A) A screen for sexual transformers. $\mathrm{F}_{1}$ animals, which segregated an approximate ratio of $25 \%$ pseudomales were considered as putative sex determination mutants. $(B)$ Pseudomales have an XX karyotype. $\mathrm{F}_{2}$ pseudomales derived from a backcross to a polymorphic strain segregated three types of SSCP patterns for the X chromosome marker S105: (C) the maternal California strain, $(\mathrm{H})$ heterozygous, or $(\mathrm{W})$ the paternal Washington strain. Animals 2 and 6 are heterozygous, indicating that they are XX and therefore sexual transformers.

Four allelic transformer mutants and the Ppa-tra-1 gene are located on chromosome III

We isolated eight $P$. pacificus transformer mutants. Mapping experiments indicated that the four mutations tu187, tu306, tu307, and tu308 are located on linkage group III, and complementation tests revealed that these mutants are allelic to one another (data not shown). Previous studies have shown that chromosome III of $P$. pacificus is syntenic to C. elegans chromosome III, although multiple independent intrachromosomal rearrangements changed the exact order of genes (Lee et al. 2003). Among the three C. elegans tra mutants, the terminal sex determination gene Cel-tra-1 is located on chromosome III. Thus, Ppa-tra-1 would be a candidate gene for the four transformer mutations of $P$. pacificus.

To determine whether a Ppa-tra-1 ortholog exists, and whether this is located on chromosome III, we used a PCR approach with degenerate primers against the zinc- 
finger region of tra-1 (see Materials and Methods). We isolated a PCR fragment that corresponds to the region of the second to the fourth finger. Conceptual translation of this region shows high amino acid similarity to CelTRA-1 (Fig. 3A). To obtain genomic sequence at the Ppatra-1 locus, the PCR fragment was used for hybridization of a $P$. pacificus BAC library. On the basis of the identi- fied BAC clones and their end sequences, different primer pairs were designed and screened for polymorphic PCR fragments. A polymorphic marker S182 was detected in the neighborhood of Ppa-tra-1 and was used to map this region onto the genetic linkage map. Linkage analysis assigned $\mathrm{S} 182$ to $P$. pacificus chromosome III between S52 and S1 (Fig. 3B).
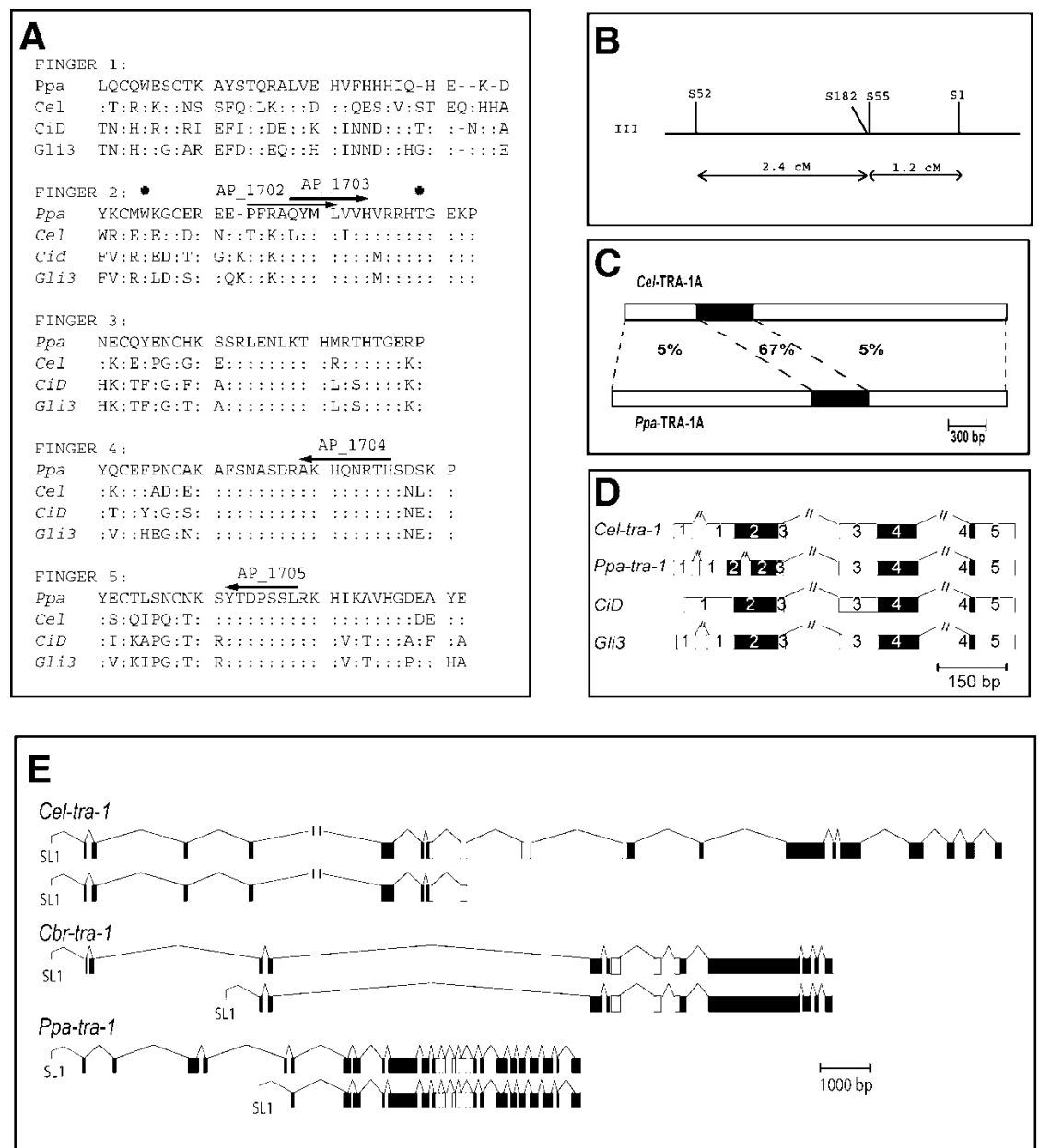

\section{$\mathbf{F}$}

MEKEDEESIE VGRSSENTDS TGSSNSIDSN VSTSLVIEZI SPPNSSISST TWNSSIPSSN ILPSSLSDVZ PIQPELTPMI SPNRNESTPK RRKRNINSEG JNGECIKRDR VMIAQSNQZY WSPFYSPSDS SFLAYCSMIYY TQSRYYSSRV CLDIDKLPFT

FZQRAHEKES LALLMSGGNG SHGVGNGQPH NCNMMVQHHQ FMEMVRRFFG MGMGLLGMOM AEMGMQITFF APSPTALAAQ

MSAMYPSSPG PSMFHPMGVQ LANGLTQSSQ MSPDEQPDIP LEQADFRIIN GYLSKWVVRV LGKVHPQDLL NVDPKVNILP

ITQNEYGNAL DFUMCFYMFV ITNHESTDGY LWFRENL_VT LELHLATTNLV NLLKSLPRGM PVPSYLRLPF NPKRKYTTVP

GVCPYGKTFO QRFEAYIDQG FNNLLLNRLKK GVPIIYPYYY DPQYDDCPGG SNEMSLPPSI PRREERDNGA PDLSPHEDMD

GGDEDREESM EDSGTUSUNS IVRFNESSHS NLPQIVMKPQ OYAKVGRRPRG RPLGSTKKIG IMGVPKGISR FERPDCYEER DAGSEDSEDI DVETLETZQC QWESCTKAYS TQRALVEHVE HHHIQHEKDY KCMUMGGCERE EPFRAQYMLV VHVRRHI)GEK FNWCQYENCH KSYSRLENLK THMRTHTGER PYQCEFPNCA KAFSNASDRA KHQNRTHSDS KPYECTLSNC NKSYTDESSL RXHIKTVHGD EAYEKTKKNK PALPPGRRRH KLPIATQIQL GQLVSSTKGQ PNKYYNPQRY EEEQRRQEAR DRAVREGNPY JAPAPAHYGV VGGGGGSTTT SPTGTTSSNG SSVHISSPZA NDSPPVNNGG GNESIEGRFL PNOQNRONRO MDVSGWLDEE KRGJDSSDDM DSTKSLIEME NHSNFLAFAN APKVDEAVZL SLIEPEVENV RDEEENDDLY GMNRREEEEG DENGEVQVMS IPLLPSRRFR MANKTIGNPR MILSLHEMNE GREIRDMNDV LNGLYEDDEN JRRRERLEEW RDEEEEEDDE NGNEEMENDH HPMEYDISTV DFSLYGGGQM PILTGEDIDE AVLSQPLSSH YSSSIPLQSS PIDLDLELID SLEMNVLVDS IEEKVUNERRR LQ
Figure 3. Molecular characterization of Ppa-tra-1. (A) The zinc-finger domain of Ppa-tra-1, Cel-tra-1, Drosophila cubitus interruptus (CiD), and human GLI3 is highly conserved. Degenerated primers that were used to clone Ppa-tra-1 are indicated by arrows. (B) Map position of Ppa-tra-1. The marker S182, which is in the BAC containing the Ppa-tra-1 gene (BAC 4E3.y), maps on chromosome III between S52 and S1 and is close to S55. (C) Cel-TRA-1A and PpaTRA-1A conservation is limited to the zinc-finger domain. Note that this domain in Ppa-TRA-1A is shifted toward C-terminal relative to Cel-TRA-1A. (D) The exonintron structure of the zinc-finger domain is conserved. The five zinc fingers are numbered. Introns are not drawn to scale. (E) Genomic structure of tra-1 in C. elegans, $C$. briggsae, and $P$. pacificus. The exons coding for the zinc fingers are in white. Alternative start codons exist in $C$. briggsae and $P$. pacificus, but not in $C$. elegans. $(F)$ Conceptual translation of Ppa-tra-1. The start methionine $(\mathrm{M})$ used for alternative splicing is indicated by an arrow, mutations are indicated by circles, and the zincfinger domain is underlined. tu306 contains a guanine-to-adenine transition and tu187 a cytosine-to-thymidine transition, resulting in a stop codon and a missense mutation, respectively. Note that the mutations are in highly conserved amino acids in finger $2(A$, asterisks). 
Sequence similarity of the nematode tra-1 gene is restricted to the zinc-finger domain

To study the structure of the Ppa-tra-1 gene, we performed RACE experiments and amplified the complete $5^{\prime}$ and $3^{\prime}$ ends of the gene. Two different transcripts were identified by $5^{\prime}$ RACE. Both transcripts are trans-spliced to the SL1 splice leader, a modification at the 5' end of messenger RNAs that is commonly seen in nematodes (Fig. 3E,F; Lee and Sommer 2003). The product of the larger transcript, designated $P p a-T R A 1 A$, is predicted to encode a protein of 1122 amino acids (Fig. 3F). The predicted product of the second transcript, Ppa-TRA-1B, is an N-terminally truncated version of Ppa-TRA-1A of 948 amino acids. Interestingly, two different transcripts with an $\mathrm{N}$-terminally truncated version are also known from the tra-1 gene of C. briggsae (de Bono and Hodgkin 1996). In contrast, the Cel-tra-1 gene has two variants with different C-terminal ends (Fig. 3E; Zarkower and Hodgkin 1992).

Sequence comparison between Cel-tra-1 and Ppa-tra-1 revealed that tra-1 is a fast evolving gene. The overall amino acid identity is $9 \%$, and the sequence similarity outside of the zinc-finger domain is only $5 \%$ (Fig 3C). Cel-TRA-1 sequences located outside of the zinc finger have been implicated in protein-protein interactions, such as the CR2 region in the $\mathrm{N}$ terminus (de Bono et al. 1995; de Bono and Hodgkin, 1996) and CR7, which binds to Cel-TRA-2 (Lum et al. 2000; Wang and Kimble 2001). However, no sequences similar to CR2 and CR7 could be identified in Ppa-TRA-1. In contrast, the zinc-finger domain of Ppa-TRA-1 shows an amino acid identity of $67 \%$ to Cel-TRA-1 and $63 \%$ to CiD and GLI3, which are other members of the GLI family of $\mathrm{C} 2 \mathrm{H} 2$ zinc-finger genes. Comparison of other zinc-finger transcription factors among insects and vertebrates has indicated that sequence similarities of about $60 \%-70 \%$ within the DNAbinding domain are common among true orthologs (Sommer et al. 1992).

The genomic structure of tra- 1 encompasses around 22 $\mathrm{kb}$ in C. elegans, $18 \mathrm{~kb}$ in C. briggsae, and $15 \mathrm{~kb}$ in $P$. pacificus. Ppa-tra-1 contains 22 introns, whereas Celtra-1 and Cbr-tra-1 contain 17 and 11 introns, respectively. Although Ppa-tra-1 has more introns than the tra-1 genes of the Caenorhabditis species, the Ppa-tra-1 gene is substantially smaller on the basis of the small size of the P. pacificus introns (Fig 3E). Strikingly, there is a high conservation of intron/exon boundaries in the zinc-finger domain among genes of the GLI family; in vertebrates, Drosophila, and nematodes, intron positions are conserved between GLI family genes (Fig. 3D).

Mutations in Ppa-tra-1 cause the transformer phenotype of tu187 and tu306

The two mutations analyzed, tu187 and tu306, show $100 \%$ linkage to the SSCP marker S182, which is located on the BAC containing the Ppa-tra-1 gene. More specifically, all pseudomales $(n=61$ for tu187 and $n=42$ for tu306) derived from a backcross to the Washington strain segregated the California SSCP pattern. This finding suggests that mutations in the Ppa-tra-1 gene might cause the observed transformer phenotype (Fig. 3B). We sequenced Ppa-tra-1 in these transformer mutants and indeed identified mutations. tu187 results in a missense mutation in the first amino acid of the linker sequence between zinc fingers 2 and 3, causing a T156I change (Fig. 3A,F). The linker sequence TGEKP is conserved among all TRA-1 orthologs, and many other zinc-finger transcription factors and mutations in this region have been shown to affect the DNA-binding affinity (Choo and Klug 1993; Clemens et al. 1994). In tu306, a nonsense mutation results in a premature stop of Ppa-tra-1 at W150, causing a protein truncation at the beginning of the second zinc finger. Together, these findings suggest that mutations in Ppa-tra-1 cause the transformer phenotype of tu187 and tu306.

\section{A Ppa-tra-1 morpholino phenocopies Ppa-tra-1 mutants}

To confirm that mutations in the Ppa-tra-1 gene cause a transformer phenotype, we developed a knockdown approach to reduce tra-1 function in $P$. pacificus. As several attempts to establish systemic RNA interference were unsuccessful in this nematode, we injected antisense morpholino oligonucleotides (MO) into wild-type $P$. pacificus hermaphrodites. Morpholinos are synthetic DNA analogs that have been shown to efficiently block translation of their target RNAs in a variety of animals (Heasman 2002). A Ppa-tra-1(MO) containing the translation start site of Ppa-tra-1 phenocopied the Ppa-tra-1 mutants (Fig. 4D). Injection of Ppa-tra-1(MO) resulted in $7 \%(n=47 / 674)$ of transformed $F_{1}$ progeny. Except for two intersexual animals (hermaphroditic tail, no vulva, and monodelphic gonad), the somatic tissues of all pseudomales were indistinguishable from wild-type males (Table 1; Fig. 4D). Using molecular markers, we confirmed that the Ppa-tra-1(MO) pseudomales indeed have an XX karyotype (data not shown).

\section{Ppa-tra-1 show soma and germ line phenotypes}

To determine the exact role of Ppa-tra-1 during sex determination, we investigated the phenotype of Ppa-tra-1 mutant animals in more detail. All four alleles are similar in phenotype, as are the most extreme morpholinotreated animals. Hermaphrodites heterozygous for Ppatra-1 mutations segregate $\sim 25 \%$ pseudomales (tu187, $n=146 / 664)$, indicating that they are recessive. In contrast to C. elegans, in which many tra-1 intersexual animals were found, all $P$. pacificus tra-1 mutants were apparently fully transformed in the soma; pseudomales contain spicules and genital papillae in the tail (Table 1; Fig. 4C,D), a monodelphic gonad, and diagonal tail muscles (Table 1; Fig. 4J). At the same time, prominent hermaphroditic structures, such as vulva and the expression of vitellogenins (Table 1; Fig. $4 \mathrm{~K}$ ), are absent.

Although the somatic transformation is nearly complete, none of the Ppa-tra-1 mutants showed normal 

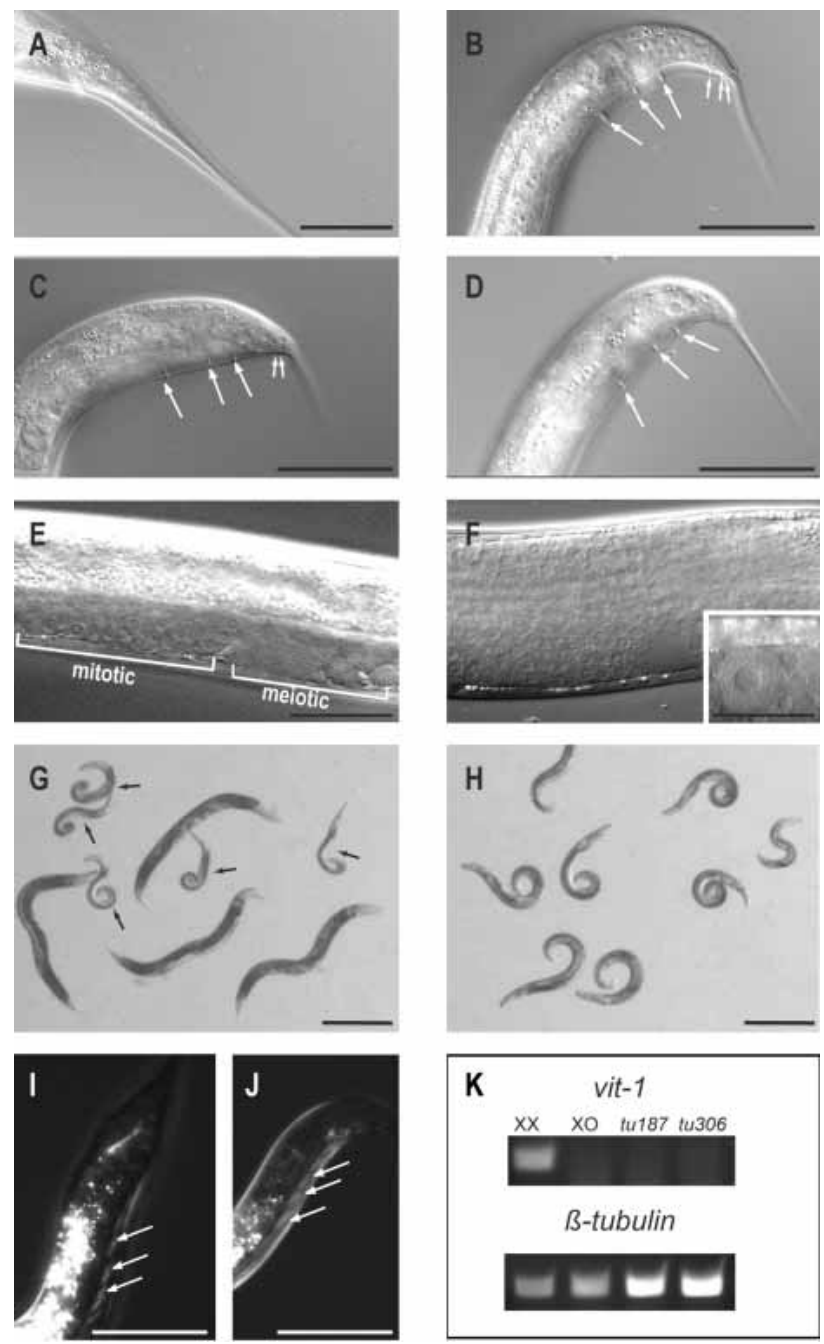

Figure 4. P. pacificus tra-1 mutant phenotype. $(A-F)$ Normarski and $(I-J)$ polarized light-optical photomicrographs. Bars, $A-F, I$, and $I, 30 \mu \mathrm{m}$. (A) Wild-type hermaphrodite tail. (B) Wildtype male tail. The broader shape, the presence of six pairs of V-rays (three of them indicated by arrows, others in a different focal plane) and three pairs of T-rays (smaller arrows) are hallmarks of male tails. (C) Ppa-tra-1(tu187) pseudomale tail. (D) Ppa-tra-1(MO). T-rays are in a different focal plane. (E) Germ line of a wild-type male. Mitotic germ cells are characterized by a round morphology and a prominent nucleolus. $(F)$ The germ line of Ppa-tra-1 (tu187) is tumorogenic and contains some oocyte-like cells (inset). $(G, H)$ Lateral view of animals in $20 \mathrm{mM}$ serotonin. Bars, $250 \mu \mathrm{m}$. (G) Serotonin elicit curling of the tail and posterior body in wild-type males (arrows) but not in hermaphrodites. (H) Ppa-tra-1(tu187) pseudomales also curl after serotonin treatment. (I) Males contain male-specific diagonal ventral muscle cells in the tail that can be visualized under polarized light. (J) Ppa-tra-1 pseudomales also develop these muscles. (K) vitelogenin-1 (vit-1) RNA, a precursor of yolk protein, is present in hermaphrodites $(\mathrm{XX})$, but not in wild-type males (XO) or any of the two tested Ppa-tra-1 mutants. Amplification of $\beta$-tubulin cDNA was used as a positive control.

mating behavior. Pseudomales of all four Ppa-tra-1 alleles and the Ppa-tra-1(MO)-treated animals produced sperm, but were not attracted by hermaphrodites and did not mate. To investigate the masculinization in the nervous system, we tested the reaction of males and pseudomales to serotonin. Serotonin is produced in male-specific neurons in the tail of various nematodes, and is important for mating behavior (Loer and Kenyon 1993). Pseudomales of all Ppa-tra-1 alleles and Ppa-tra-1(MO) animals contracted their tails when incubated in serotonin, similar to wild-type males (Table 1; Fig. 4G,H). This result indicates that the motor neurons inervating the ventral tail muscles are also transformed. Unfortunately, other staining protocols for specific C. elegans neurons cannot be transferred to $P$. pacificus. Therefore, it remains unknown whether other types of male-specific neurons are missing in Ppa-tra-1 pseudomales.

In contrast to the soma, transformation in the germ line is frequently incomplete in Ppa-tra-1 pseudomales, and the phenotype differs between the Ppa-tra-1 alleles. A variable number of oocytes (beween 1 and 8 per animal) was found in Ppa-tra-1 mutant and Ppa-tra-1(MO) animals (Table 1; Fig. 4F). In particular, $77 \%$ of Ppa-tra1(tu306) mutant animals, but only $7 \%$ of the Ppa-tra1(MO) animals contained oocytes (Table 1). Cel-tra-1 mutant animals also have a germ line phenotype. Most Cel-tra-1 alleles show an incomplete germ line transformation and are characterized by the presence of some oocytes and reduced sperm production when compared with wild-types males (Schedl et al. 1989). Ppa-tra-1 mutants have a second phenotype in the germ line, which is unknown from C. elegans. In Ppa-tra-1(tu187) and Ppatra-1(tu308), $61 \%$ and $47 \%$ of the analyzed mutant animals, respectively, showed an overproliferation of the germ line (Table 1; Fig. 4F). In contrast, in Ppa-tra1(tu306) mutant and Ppa-tra-1(MO) animals, only a small number of animals showed germ line overproliferation. These results might provide a first indication that the morpholino technology works less efficient in the germ line, as both the presence of oocytes and the overproliferation are observed with a lower frequency in morpholino-treated animals.

To investigate the phenotype of Ppa-tra-1 mutants in X0 males, we crossed tu187 to ped-14 (tu114). ped-14 has a high incidence of male (him) phenotype and is characterized by the expression of a dumpy phenotype in XX animals, but not in XO animals. In Ppa-tra-1 (tu187); ped-14 double mutants, the soma of all non-dumpy (XO) males was indistinguishable from wild-type males (Fig. 4C). We therefore concluded that Ppa-tra-1 is dispensable for the generation of males. Taken together, the genetic, phenotypic, and morpholino analyses suggest that Ppa-tra-1 is a global regulator of sex determination in $P$. pacificus. Ppa-tra-1 and Cel-tra-1 show only limited sequence similarity, indicating that the genes are fast evolving. Nonetheless, they are true orthologs with a conserved function in nematode sex determination.

\section{Discussion}

Several studies on sex determination in animals have indicated substantial differences between species, often among close relatives (Zarkower 2001). In addition, com- 
Table 1. Phenotype of Ppa-tra-1 mutant alleles and the Ppa-tra-1(MO) phenocopy (\%)

\begin{tabular}{lcccccrrr}
\hline & $\begin{array}{c}\text { Presence } \\
\text { of rays }\end{array}$ & Vulva & $\begin{array}{c}\text { Monodelphic } \\
\text { gonad }\end{array}$ & $\begin{array}{c}\text { Presence } \\
\text { of spicules }\end{array}$ & $\begin{array}{c}\text { Deformed } \\
\text { spicules }\end{array}$ & $\begin{array}{c}\text { Tumorous } \\
\text { Oocytes }\end{array}$ & $\begin{array}{c}\text { Curling in } \\
\text { serotonin }\end{array}$ \\
\hline tu187 $(n=36)$ & 100 & 0 & 100 & 100 & 0 & 38 & 61 & 92 \\
tu306 $(n=35)$ & 100 & 0 & 100 & 100 & 0 & 77 & 5 & 78 \\
tu307 $(n=21)$ & 100 & 0 & 100 & 100 & 0 & 14 & 24 & 90 \\
tu308 $(n=34)$ & 100 & 0 & 100 & 100 & 11 & 26 & 47 & 71 \\
Ppa-tra-1 $(M O)(n=27)$ & 100 & 0 & 100 & 100 & 0 & 7 & 11 & 100 \\
\hline
\end{tabular}

parative studies between members of the same genus, such as nematodes of the genus Caenorhabdits, provided clear evidence for the rapid evolution of sex determination genes. To study the evolution of sex determination pathways and genes in an unbiased way, we have started to use a forward genetic approach. The nematode $P$. pacificus has been developed as a satellite organism and provides genetic, genomic, and molecular tools to investigate evolutionary questions. A comparison of sex determination between two distantly related species of the same phylum, such as $P$. pacificus and C. elegans, can help us in understanding how this process evolved. In the long run, such a comparison can also provide an insight into the evolution of signaling pathways per se (Pires-daSilva and Sommer 2003). Here, we report the functional conservation of the tra-1 gene in sex determination of $P$. pacificus and C. elegans. As these two nematodes shared a last common ancestor 200-300 mya, this ancestor must have used a homologous TRA-1 protein for sex determination. Our studies provide the first example of the functional conservation of a global sex determination regulator in two distantly related species of the same phylum.

tra-1 loss-of-function mutations result in a hermaphrodite-to-male sex reversal in both $P$. pacificus and $C$. elegans. Furthermore, knock down of Ppa-tra-1 using morpholino oligonucleotides induces the same kind of sex reversal. These results indicate that TRA-1 function is required for promoting hermaphroditic development in both species. Several aspects of TRA-1 sequence and function are of evolutionary importance. The similarity of TRA-1 function does not correlate with the overall sequence conservation, a pattern that had so far only been observed in more closely related species. This finding suggests important functional constraints at the transcriptional level. Although the overall sequence similarity between Ppa-TRA-1 and Cel-TRA-1 is very low, sequence identity in the zinc-finger domains remains with $67 \%$ very high. This additionally supports the idea that the transcriptional activity of TRA-1 is evolutionarily constrained. Also, mutations found in Ppatra-1 are predicted to disrupt the zinc finger or to affect DNA binding. The zinc-finger domain of TRA-1 has been primarily implicated in DNA binding to regulate the transcription of target genes. However, more recently, this domain has been shown to also bind RNA (Graves et al. 1999). Most likely, the zinc-finger domain is required for regulating several downstream target genes. In C. elegans, tra-1 was shown to regulate down- stream genes in a tissue-specific manner, egl-1 in HSN neurons (Conradt and Horvitz 1999), fog-3 in the germ line (Chen and Ellis 2000), and mab-3 in the intestine, tail, and neurons (Yi et al. 2000). These observations support the hypothesis that changes in the zinc-finger domain and the function of the protein are evolutionary constrained. In contrast, the additional conservation of the splicing pattern in the zinc-finger domain between vertebrates, insects, and nematodes cannot be explained by functional constraints. One could speculate however, that alternative splicing such as seen in the case of Celtra- $1 B$, is a common and functionally important phenomenon of GLI/CiD genes that contributes to the conservation of splice sites.

The lack of sequence conservation in other regions of Cel-TRA-1, such as CR2 and CR7, may be the consequence of coevolution between interacting partners. Consistent with this, the CR7 region of TRA-1 in C. elegans and $C$. briggsae has been shown to interact with TRA-2 in a strictly species-specific manner (Wang and Kimble 2001). Compensatory mutations in both genes were therefore required to maintain the binding abilities in their products.

GLI/CiD transcription factors are components of the Hedgehog-signaling pathway in most organisms (PiresdaSilva and Sommer 2003). In C. elegans, many components of the canonical Hedgehog signaling pathway are absent, and the GLI-like transcription factor TRA-1 has acquired a novel function in sex determination. Our finding of Ppa-TRA-1 function during sex determination might represent a first indication that the loss of the Hedgehog signaling pathway occurred early in nematode evolution. It should be noted that we have identified in a BAC end and fosmid end-sequencing project, remnants of two additional GLI-like transcription factors. However, we have so far not been able to detect corresponding cDNA fragments, so that it remains unknown whether these DNA fragments belong to real genes rather than to pseudogenes (A. Pires-daSilva and R.J. Sommer, unpubl.).

The functional conservation of TRA-1 is extremely interesting considering the position of TRA-1 in the sex determination hierarchy. tra-1 is the most downstream global regulator of sex determination in C. elegans. Global regulators can act as a switch of the sexual fate of the complete organism, whereas local regulators only control the sexual fate of individual cells or tissues. In addition to being the most downstream global regulator of sex determination, tra-1 is the only globally acting mol- 
ecule that is involved in transcriptional regulation. The more upstream part of the sex determination hierarchy is involved in transducing the primary $\mathrm{X}$ :A ratio and the regulation of TRA-1 activity. Therefore, conservation of TRA-1 function between $P$. pacificus and C. elegans is most informative with regard to the transcriptional machinery acting downstream of tra-1. It remains unknown, however, whether the more upstream factors are also conserved. In principle, TRA-2 and TRA-3 might constitute an ancestral system that coevolved within nematodes. Fast coevolution between interacting proteins has been reported for TRA-1 and TRA-2, which interact in a species-specific manner in C. elegans and $C$. briggsae (Wang and Kimble 2001). Alternatively, the regulation of TRA-1 activity could be achieved by molecular mechanisms other than a TRA-2/TRA-3 system, which regulates nuclear entry of TRA-1. For example, TRA-1 activity might be regulated by phosphorylation by some other signaling pathway in other nematode species, such as $P$. pacificus. Inspired by the finding of rapid evolutionary changes of sex determination systems, several authors have discussed sex determination as a model to understand the evolution of signaling pathways (Wilkins 2002). Several theoretical studies suggested that such pathways arise by retrograde evolution from bottom to top, with a potential loss (shrinkage) and regain (growth) of genes.

The conservation of TRA-1 between $P$. pacificus and C. elegans is consistent with the retrograde evolution model. As discussed above, TRA-1 activity itself might be regulated in different ways in $P$. pacificus and $C$. elegans. For example, a potential role for TRA-1 phosphorylation might have evolved in response to the loss of upstream components of an ancestral pathway. Genetic and molecular analysis of other transformer mutations in $P$. pacificus might shed light onto these questions. Considering that some of the other sex determination genes are even more diverged between C. elegans and $C$. briggsae than tra-1, gene predictions and candidate gene approaches are unlikely to provide further molecular understanding of sex determination in P. pacificus. The recently developed genome map of $P$. pacificus provides a unique setting for positional cloning, and therefore, makes forward genetic approaches feasible (Srinivasan et al. 2002, 2003). Complementing the forward genetic approach with yeast two-hybrid studies, a more extensive comparison between these two nematode species can elucidate the molecular mechanisms underlying the evolutionary dynamics of sex determination.

\section{Materials and methods}

Nematode strains and cultures

Worms were grown on Escherichia coli OP50 bacterial lawn, as described elsewhere (Sommer and Sternberg 1996). The following strains were used in this study: $P$. pacificus PS312 (the wildtype strain), a derivative of an isolate from Pasadena, California; P. pacificus PS1843, isolated from Port Angeles, Washington (Srinivasan et al. 2001). P. pacificus mutants are as follows: unc-14(sy361), an uncoordinated X-linked mutant (Sommer et al. 1996); ped-14(tu114), a mutant originally identified for its egg-laying defective (egl) phenotype (A. Pires-daSilva and R.J. Sommer, unpubl.); and dpy-1(sy342), a dumpy mutant located on chromosome III that was used as balancer for tra-1 mutation.

\section{Mutagenesis}

Mixed stage animals of $P$. pacificus PS312 (California) were washed off the plates in M9 buffer, and ethyl methanesulphonate (EMS) added to a final concentration of $50 \mathrm{mM}$ for $4 \mathrm{~h}$ at $20^{\circ} \mathrm{C}$. The suspension was washed in M9 five times, and the worms were spotted onto the surface of NG plates (Wood 1988). After $1 \mathrm{~h}$, individual motile L4 hermaphrodites were picked individually to plates. Subsequently, $10 \mathrm{~F}_{1}$ progeny per mutagenized animal were picked to single plates and after 4-5 d, the $\mathrm{F}_{2}$ were screened for mutant phenotypes. In plates where pseudomales were observed, about 10 sister hermaphrodites were picked for maintenance of heterozygous mutants. Mutant hermaphrodites were back-crossed at least three times using PS312 wild-type males.

\section{PCR cloning of Ppa-tra-1}

Degenerate oligonucleotides were designed to amplify zinc fingers 2-4, on the basis of the sequence alignment with C. elegans tra-1, Drosophila cubitus interruptus Dominant (CiD), and human GLI3 (Zarkower and Hodgkin 1992). Nested reverse transcription PCR (RT-PCR) using $P$. pacificus mixed-stage cDNA as template was performed with primers AP_1702 and AP_1705 for the first round, and AP_1703 and AP_1704 for the second round. PCR reactions consisted of $10 \mathrm{mM}$ Tris- $\mathrm{HCl}, 50 \mathrm{mM}$ $\mathrm{KCl}, 1.5 \mathrm{mM} \mathrm{MgCl}_{2}, 200 \mathrm{mM} \mathrm{dNTP}, 1 \mathrm{U}$ Taq polymerase, and $1 \mathrm{mM}$ of each primer. Thermocycling was done in a Perkin Elmer Gene Amp 9700 PCR machine under conditions consisting of an initial denaturation at $94^{\circ} \mathrm{C}$ for $3 \mathrm{~min}$, followed by 30 cycles of $94^{\circ} \mathrm{C}$ for $1 \mathrm{~min}, 50^{\circ} \mathrm{C}$ for $1 \mathrm{~min}$, and $72^{\circ} \mathrm{C}$ for $1 \mathrm{~min}$, and a final incubation at $72^{\circ} \mathrm{C}$ for $7 \mathrm{~min}$. PCR fragments of the expected size (213 bp) were cloned into TOPO vector (Invitrogen) and sequenced according to manufacturer's instructions for the Big Dye Terminator chemistry (Applied Biosystems). New primers were designed to amplify the $5^{\prime}$ and $3^{\prime}$ regions of $P p a$ tra-1 using RACE (rapid amplification of cDNA ends) experiments (Frohman 1993). The Genbank accession number for Ppatra-1 is AY579380.

\section{Oligonucleotides}

AP_1702, 5'-CCNTTYAARGCNYWNTAKATGYT-3'; AP_1703, 5'-CARTAYATGYTNGTNGTNCA-3'; AP_1704, 5' -TGNGTNC KRTTYTGRTGYTTNGC-3'; AP_1705， 5'-ARNGWNGWNG GRTCNGTRTA-3'; AP_1925， 5'-AAGTTTCCCCAGACCTG GAA-3'; AP_1926, 5'-GGGAATGAATCCATTGGAGA-3'; tra-1_ATG (MO), 5'-CATTACCCCCCGACATTAGCAGCG C-3'; vab-7_ATG (MO), 5'-AGGGTTGCATCGGATGGCC GGGCAT-3'; AP_3589, 5'-GGATATCGAGGAGTTCCATCG C-3'; AP_3590, 5'-GGAGATTCACGAAGAAGACGATGG-3'; AG_2932, 5'-CAACGTTTACTACAATGAGG-3'; and AG_2933, 5'-TTGCGAAGATCAGCATTGAG-3'.

\section{Polymorphic marker close to tra-1}

To obtain genomic sequence close to the Ppa-tra-1 locus, a radiolabeled cDNA fragment containing the zinc-finger domain 
was hybridized to a filter containing the $P$. pacificus HindIIIBAC (Bacteria Artificial Chromosome) library (Srinivasan et al. 2002) under the following conditions: $200 \mathrm{mM}$ sodium-phosphate buffer ( $\mathrm{pH} 7.2) ; 1 \mathrm{mM}$ EDTA; $15 \%$ formamide; $1 \%$ BSA; $7 \%$ SDS for $16 \mathrm{~h}$ at $42^{\circ} \mathrm{C}$, washed three times with $0.2 \times$ SSC and $0.1 \%$ SDS at $60^{\circ} \mathrm{C}, 20 \mathrm{~min}$ each. In total, 10 distinct BAC clones hybridized to this probe. Sequences obtained from the BAC ends of those identified BACs were used to design PCR primers that amplify DNA fragments of about $200 \mathrm{bp}$. A PCR fragment generated with the primer pair AP_1925 and AP_1926, based on the BAC end BACPP28-N18.z, showed mobility differences in a SSCP gel between reactions using genomic DNA templates of $P$. pacificus PS312 (California) and P. pacificus PS1843 (Washington). This primer pair was designated as SSCP marker S182.

\section{Gel electrophoresis and SSCP detection}

For SSCP detection, PCR samples were diluted 1:1 in denaturing solution $(95 \%$ formamide, $0.1 \%$ xylene cyanol, $0.1 \%$ bromophenol blue), denatured at $95^{\circ} \mathrm{C}$ for $5 \mathrm{~min}$, and loaded onto a GeneGel Excel prepoured 6\% acrylamide gel (PharmaciaBiotech). Gels were fixed and silver stained to detect the DNA.

\section{PCR amplification and mapping}

For mapping, heterozygous mutant hermaphrodites in the California background were crossed with males of the Washington strain. To extract genomic DNA, $\mathrm{F}_{2}$ mutant pseudomales were picked to single tubes containing $2.5 \mu \mathrm{L}$ of lysis buffer $(50 \mathrm{mM}$ $\mathrm{KCl}, 10 \mathrm{mM}$ Tris- $\mathrm{HCl}$ at $\mathrm{pH} 8.3,2.5 \mathrm{mM} \mathrm{MgCl}, 0.45 \% \mathrm{NP}-40$, $0.45 \%$ Tween, $0.01 \%$ gelatin, $5 \mu \mathrm{g} / \mathrm{mL}$ Proteinase $\mathrm{K}$ ) and incubated for $1 \mathrm{~h}$ at $65^{\circ} \mathrm{C}$, followed by inactivation of the Proteinase $\mathrm{K}$ at $95^{\circ} \mathrm{C}$ for $10 \mathrm{~min}$. To assign linkage of a mutation to a certain chromosome, two representative SSCP markers per chromosome were tested against 21 Washington-back-crossed mutant animals. We used the following SSCP markers for chromosome assignment: chromosome I: S28, S31; chromosome II: S38, S77; chromosome III: S52, S55; chromosome IV: S34, S148; chromosome V: S106, S142; and chromosome X: S105, S140. For a typical PCR assay, DNA derived from one worm was used as a template for two distinct PCR reactions. In one of them, we determine whether the mutants were heterozygous for the $\mathrm{X}$ chromosome markers. If this was the case, those animals were used for further mapping studies in a second PCR reaction. The PCR conditions were the same as described above.

\section{Complementation tests}

Complementation tests were carried out by generating a hermaphroditic strain phenotypically marked with the X-linked mutation unc-14 together with the transformer mutation, that is, tu187, balanced by the $d p y-1$ mutation (unc-14; tu187 +/+ d1). Hermaphrodites of this strain were crossed with males heterozygous for a transformer mutation. In the $\mathrm{F}_{1}$ generation, the unc-14 mutation is used to distinguish $\mathrm{XO}$ male self-progeny, $\mathrm{XX}$ pseudomale self-progeny, and XO male cross-progeny (all with unc phenotype) from XX pseudomale cross-progeny.

\section{Morpholino injections}

The morpholino oligonucleotide tra-1_ATG (Gene Tools) was dissolved in water and subsequently diluted in $1 \times$ injection buffer [20\% polyethylene glycol, MW 6000-8000; 200 mM potassium phosphate $(\mathrm{pH} 7.5) ; 30 \mathrm{mM}$ potassium citrate $(\mathrm{pH} 7.5)]$ to a concentration of $100 \mu \mathrm{M} . \mathrm{F}_{1}$ hermaphrodites derived from a cross between $P$. pacificus California and Washington strains were injected in the distal arm of the gonad. The phenotypic effects of the morpholino were scored in animals of the next generation 3-4 d later. Pseudomales were picked to single tubes and processed for DNA extraction and SSCP marker detection for the X-chromosome as described above (PCR amplification and mapping). Injections of hermaphrodites with morpholinos targeting Ppa-vab-7 (oligonucleotide vab-7_ATG) did not result in transformers in the $F_{1}$ generation.

\section{RT-PCR for vitellogenin-1}

Pools of 10 worms were picked into a microfuge containing 10 $\mu \mathrm{L}$ of water and $200 \mu \mathrm{L}$ of TRIzol reagent (Life Technologies). After freezing at $-80^{\circ} \mathrm{C}$ for $15 \mathrm{~min}$, the worms were thawed and sonicated for $1 \mathrm{~min}$. Subsequently, the RNA was extracted according to the manufacturer's protocol (Life Technologies) and resuspended in $25 \mu \mathrm{L}$ of water. Subsequently, the RNA was DNase-treated with the DNA-free kit (Ambion). An 8- $\mu \mathrm{L}$ aliquot from each sample was used for reverse transcription using SuperscriptII Reverse Transcriptase (Life Technologies) and random hexamers in a $20-\mu \mathrm{L}$ volume. A total of $2 \mu \mathrm{L}$ of each reaction was taken for PCR to amplify Ppa-vit-1 (primers AP_3589 and AP_3590) and $\beta$-tubulin (AG_2932 and AG_2933). Samples were amplified for $35 \mathrm{cycles}\left(30 \mathrm{sec}\right.$ at $94^{\circ} \mathrm{C}, 30 \mathrm{sec}$ at $55^{\circ} \mathrm{C}$, and $30 \mathrm{sec}$ at $\left.72^{\circ} \mathrm{C}\right)$.

\section{General DNA analysis}

General DNA manipulation and analysis were done using standard protocols described by Sambrook and Russell (2001).

\section{Phylogenetic relationship between $\mathrm{P}$. pacificus and C. elegans}

In nematodes, no fossil record exists, so that phylogenetic separation time estimates are based solely on molecular data. Previously, the separation time for Caenorhabditis and Pristionchus has been estimated to be $\sim 100-200$ million years (Eizinger and Sommer 1997). New evidence based on genome sequencing of C. elegans and C. briggsae suggests evolutionary separation times of $\sim 100$ million years for these two Caenorhabditis species (Coghlan and Wolfe 2002; Stein et al. 2003). C. elegans and C. briggsae are two of the most closely related species of the genus, which consists of at least 18 species with much deeper phylogenetic branches (Sudhaus and Kiontke 1996). Caenorhabditis belongs to the large family of the Rhabditidae, whereas Pristionchus belongs to the Diplogastridae family. Given the current estimates based on the C. elegans/C. briggsae genome comparison, the separation time between Caenorhabditis and Pristionchus has been accordingly adjusted to at least 200-300 million years.

\section{Acknowledgments}

We thank David Rudel for providing some of the tra-1 alleles, the members of the Sommer laboratory for discussions, and Drs. A. Streit and J. Srinivasan for critically reading the manuscript.

The publication costs of this article were defrayed in part by payment of page charges. This article must therefore be hereby marked "advertisement" in accordance with 18 USC section 1734 solely to indicate this fact.

\section{References}

Barolo, S. and Posakony, J.W. 2002. Three habits of highly effective signaling pathways: Principles of transcriptional con- 
trol by developmental cell signaling. Genes \& Dev. 16: 1167-1181.

Bull, J.J. 1983. Evolution of sex determining mechanisms. Benjamin/Cummings, Menlo Park, CA.

Chen, P. and Ellis, R.E. 2000. TRA-1A regulates transcription of fog-3, which controls germ cell fate in C. elegans. Development 127: 3119-3129.

Choo, Y. and Klug, A. 1993. A role in DNA binding for the linker sequences of the first three zinc fingers of TFIIIA. Nucleic Acids Res. 21: 3341-3346.

Clemens, K.R., Zhang, P., Liao, X., McBryant, S.J., Wright, P.E., and Gottesfeld, J.M. 1994. Relative contributions of the zinc fingers of transcription factor IIIA to the energetics of DNA binding. J. Mol. Biol. 244: 23-35.

Coghlan, A. and Wolfe, K.H. 2002. Fourfold faster rate of genome rearrangement in nematodes than in Drosophila. Genome Res. 12: 857-867.

Conradt, B. and Horvitz, H.R. 1999. The TRA-1A sex determination protein of $C$. elegans regulates sexually dimorphic cell deaths by repressing the egl-1 cell death activator gene. Cell 98: 317-327.

de Bono, M. and Hodgkin, J. 1996. Evolution of sex determination in Caenorhabditis: Unusually high divergence of tra-1 and its functional consequences. Genetics 144: 587-595.

de Bono, M., Zarkower, D., and Hodgkin, J. 1995. Dominant feminizing mutations implicate protein-protein interactions as the main mode of regulation of the nematode sexdetermining gene tra-1. Genes \& Dev. 9: 155-167.

Eizinger, A. and Sommer, R.J. 1997. The homeotic gene lin-39 and the evolution of nematode epidermal cell fates. Science 278: 452-455.

Ellis, R.E. and Horvitz, H.R. 1991. Two C. elegans genes control the programmed deaths of specific cells in the pharynx. Development 112: 591-603.

Ellis, R.E. and Kimble, J. 1995. The fog-3 gene and regulation of cell fate in the germ line of Caenorhabditis elegans. Genetics 139: 561-577.

Frohman, M.A. 1993. Rapid amplification of complementary DNA ends for generation of full-length complementary DNAs: Thermal RACE. Methods Enzymol. 218: 340-356.

Gerhart, J. 1999. 1998 Warkany lecture: Signaling pathways in development. Teratology 60: 226-239.

Goodwin, E.B. and Ellis, R.E. 2002. Turning Clustering loops: Sex determination in Caenorhabditis elegans. Curr. Biol. 12: R111-R120.

Graves, L.E., Segal, S., and Goodwin, E.B. 1999. TRA-1 regulates the cellular distribution of the tra-2 mRNA in C. elegans. Nature 399: 802-805.

Haag, E.S. and Kimble, J. 2000. Regulatory elements required for development of Caenorhabditis elegans hermaphrodites are conserved in the tra-2 homologue of C. remanei, a male/ female sister species. Genetics 155: 105-116.

Haag, E.S., Wang, S., and Kimble, J. 2002. Rapid coevolution of the nematode sex-determining genes fem-3 and tra-2. Curr. Biol. 12: 2035-2041.

Hansen, D. and Pilgrim, D. 1998. Molecular evolution of a sex determination protein. FEM-2 (pp2c) in Caenorhabditis. Genetics 149: 1353-1362.

Heasman, J. 2002. Morpholino oligos: Making sense of antisense? Dev. Biol. 243: 209-214.

Hediger, M., Burghardt, G., Burghardt, G., Siegenthaler, C., Buser, N., Hilfiker-Kleiner, D., Dubendorfer, A., and Bopp, D. 2004. Sex determination in Drosophila melanogaster and Musca domestica converges at the level of the terminal regulator doublesex. Dev. Genes Evol. 214: 29-42.

Hodgkin, J. 1980. More sex-determination mutants of Cae- norhabditis elegans. Genetics 96: 649-664.

Hodgkin, J.A. and Brenner, S. 1977. Mutations causing transformation of sexual phenotype in the nematode Caenorhabditis elegans. Genetics 86: 275-287.

Kuwabara, P.E. 1996. Interspecies comparison reveals evolution of control regions in the nematode sex-determining gene tra2. Genetics 144: 597-607.

Lee, K.Z., and Sommer R.J. 2003. Operon structure and transsplicing in the nematode Pristionchus pacificus. Mol. Biol. Evol. 20: 2097-2103.

Lee, K.Z., Eizinger, A., Nandakumar, R., Schuster, S.C., and Sommer, R.J. 2003. Limited microsynteny between the genomes of Pristionchus pacificus and Caenorhabditis elegans. Nucleic Acids Res. 31: 2553-2560.

Loer, C.M. and Kenyon, C.J. 1993. Serotonin-deficient mutants and male mating behavior in the nematode Caenorhabditis elegans. J. Neurosci. 13: 5407-5417.

Lum, D.H., Kuwabara, P.E., Zarkower, D., and Spence, A.M. 2000. Direct protein-protein interaction between the intracellular domain of TRA-2 and the transcription factor TRA1A modulates feminizing activity in C. elegans. Genes \& Dev. 14: 3153-3165.

Marin, I. and Baker, B.S. 1998. The evolutionary dynamics of sex determination. Science 281: 1990-1994.

Nelson, G.A., Lew, K.K., and Ward, S. 1978. Intersex, a temperature-sensitive mutant of the nematode Caenorhabditis elegans. Dev. Biol. 66: 386-409.

Pieau, C. 1996. Temperature variation and sex determination in reptiles. Bioessays 18: 19-26.

Pires-daSilva, A. and Sommer, R.J. 2003. The evolution of signalling pathways in animal development. Nat. Rev. Genet. 4: $39-49$

Raymond, C.S., Shamu, C.E., Shen, M.M., Seifert, K.J., Hirsch, B., Hodgkin, J., and Zarkower, D. 1998. Evidence for evolutionary conservation of sex-determining genes. Nature 391: 691-695.

Sambrook, J. and Russell, D.W. 2001. Molecular cloning: A laboratory manual. Cold Spring Harbor Laboratory Press, Cold Spring Harbor, NY.

Schedl, T., Graham, P.L., Barton, M.K., and Kimble, J. 1989. Analysis of the role of tra-1 in germline sex determination in the nematode Caenorhabditis elegans. Genetics 123: 755 769.

Shen, M.M. and Hodgkin, J. 1988. mab-3, a gene required for sex-specific yolk protein expression and a male-specific lineage in C. elegans. Cell 54: 1019-1031.

Sommer, R.J. 2001. As good as they get: Cells in nematode vulva development and evolution. Curr. Opin. Cell. Biol. 13: 715720.

Sommer, R.J. and Sternberg, P.W. 1996. Apoptosis and change of competence limit the size of the vulva equivalence group in Pristionchus pacificus: A genetic analysis. Curr. Biol. 6: $52-$ 59.

Sommer, R.J., Retzlaff, M., Goerlich, K., Sander, K., and Tautz, D. 1992. Evolutionary conservation pattern of zinc-finger domains of Drosophila segmentation genes. Proc. Natl. Acad. Sci. 89: 10782-10786.

Sommer, R.J., Carta, L.K., Kim, S.Y., and Sternberg, P.W. 1996. Morphological, genetic and molecular description of Pristionchus pacificus sp (Nematoda: Neodiplogastridae). Fund. Appl. Nematol. 19: 511-521.

Srinivasan, J., Pires-daSilva, A., Gutierrez, A., Zheng, M., Jungblut, B., Witte, H., Schlak, I., and Sommer, R.J. 2001. Microevolutionary analysis of the nematode genus Pristionchus suggests a recent evolution of redundant developmental mechanisms during vulva formation. Evol. Dev. 3: 229-240. 
Srinivasan, J., Sinz, W., Lanz, C., Brand, A., Nandakumar, R., Raddatz, G., Witte, H., Keller, H., Kipping, I., Pires-daSilva, A., et al. 2002. A bacterial artificial chromosome-based genetic linkage map of the nematode Pristionchus pacificus. Genetics 162: 129-134.

Srinivasan, J., Sinz, W., Jesse, T., Wiggers-Perebolte, L., Jansen, K., Buntjer, J., van der Meulen, M., and Sommer, R.J. 2003. An integrated physical and genetic map of the nematode Pristionchus pacificus. Mol. Genet. Genomics 269: 715-722.

Stein, L.D., Bao, Z., Blasiar, D., Blumenthal, T., Brent, M.R., Chen, N., Chinwalla, A., Clarke, L., Clee, C., Coghlan, A., et al. 2003. The genome sequence of Caenorhabditis briggsae: A platform for comparative genomics. PLoS Biol. 1: E45.

Stothard, P. and Pilgrim, D. 2003. Sex-determination gene and pathway evolution in nematodes. Bioessays 25: 221-231.

Stothard, P., Hansen, D., and Pilgrim, D. 2002. Evolution of the PP2C family in Caenorhabditis: Rapid divergence of the sexdetermining protein FEM-2. J. Mol. Evol. 54: 267-282.

Streit, A., Li, W., Robertson, B., Schein, J., Kamal, I.H., Marra, M., and Wood, W.B. 1999. Homologs of the Caenorhabditis elegans masculinizing gene her-1 in C. briggsae and the filarial parasite Brugia malayi. Genetics 152: 1573-1584.

Sudhaus, W. and Kiontke, K. 1996. Phylogeny of Rhabditis subgenus Caenorhabditis (Rhabditidae, Nematoda). I. Zool. Syst. Evol. Res. 34: 217-233.

Trent, C., Tsuing, N., and Horvitz, H.R. 1983. Egg-laying defective mutants of the nematode Caenorhabditis elegans. Genetics 104: 619-647.

Wang, S. and Kimble, J. 2001. The TRA-1 transcription factor binds TRA-2 to regulate sexual fates in Caenorhabditis elegans. EMBO J. 20: 1363-1372.

Wilkins, A.S. 1995. Moving up the hierarchy: A hypothesis on the evolution of a genetic sex determination pathway. Bioessays 17: 71-77.

- 2002. The evolution of developmental pathways. Sinauer Associates, Sunderland, MA.

Wood, W.B. 1988. The nematode Caenorhabditis elegans. Cold Spring Harbor Laboratory, Cold Spring Harbor, NY.

Yi, W., Ross, J.M., and Zarkower. D., 2000. mab-3 is a direct tra-1 target gene regulating diverse aspects of C. elegans male sexual development and behavior. Development 127: 4469-4480.

Zarkower, D. 2001. Establishing sexual dimorphism: Conservation amidst diversity? Nat. Rev. Genet. 2: 175-185.

Zarkower, D. and Hodgkin, J. 1992. Molecular analysis of the C. elegans sex-determining gene tra-1: A gene encoding two zinc finger proteins. Cell 70: 237-249. 


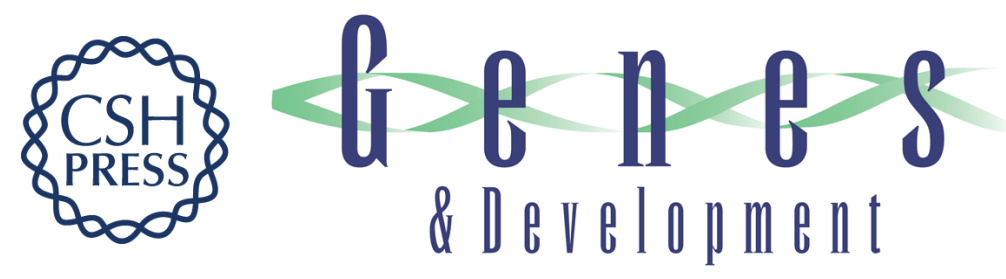

\section{Conservation of the global sex determination gene tra-1 in distantly related nematodes}

André Pires-daSilva and Ralf J. Sommer

Genes Dev. 2004, 18:

Access the most recent version at doi:10.1101/gad.293504

References This article cites 51 articles, 22 of which can be accessed free at: http://genesdev.cshlp.org/content/18/10/1198.full.html\#ref-list-1

License

Email Alerting

Receive free email alerts when new articles cite this article - sign up in the box at the top Service right corner of the article or click here.

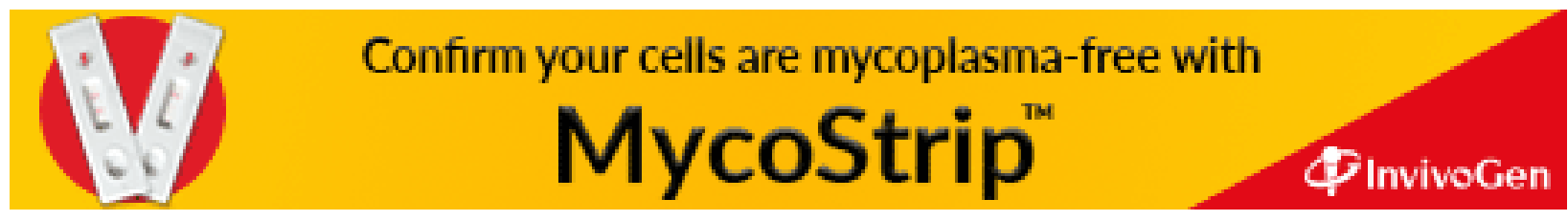

